\title{
Morphological and Molecular Characterization of Coccidiosis in Local Chickens of Mekong Delta in Vietnam
}

\author{
Hung Huu Nguyen ${ }^{1}$, Trung Van Le ${ }^{2}$, Tien Ai Lu ${ }^{1}$, and Tran Nguyen-Ho-Bao ${ }^{1 *}$ \\ ${ }^{I}$ Department of Veterinary Medicine, College of Agriculture, Can Tho University, 900000 Can Tho, Vietnam \\ ${ }^{2}$ Hau Giang Community College, Vi Thanh, 910000 Hau Giang, Vietnam \\ *Corresponding author's Email: nhbtran@ctu.edu.vn; ORCID: 0000-0002-8933-6349
}

Received: 01 November 2021

Accepted: 12 December 2021

\begin{abstract}
Eimeria species are causative pathogens considered as a tremendous threat to the poultry industry in Vietnam. Sufficient assessment of the coccidiosis prevalence is critical for improving the prophylaxis strategies to control the disease. The objective of the current study was to investigate the prevalence of coccidiosis in local chickens (Noi breed) in the Mekong Delta, Vietnam. A total of 1200 fecal samples were collected from 20 various farms in Ben Tre and Hau Giang provinces. Using a microscopic examination, the prevalence rates of coccidiosis in local chickens in Ben Tre and Hau Giang provinces were $65.83 \%$ and $68.50 \%$, respectively. Eimeria species identification was performed based on morphological characteristics and molecular methods. PCR analysis was conducted by targeting the internal transcribed spacer-1 (ITS-1) region of the Eimeria genome. Four Eimeria species, namely E. mitis, E. acervulina, E. maxima, and E. tenella were found in both methods. By applying PCR, the infection rates of E. mitis, E. acervulina, E. maxima, and E. tenella were $20 \%, 61.67 \%, 66.66 \%$, and $83.33 \%$, respectively. The findings can be considered as the first attempt to identify Eimeria species at the molecular level in local chickens (Noi breed) in the Mekong Delta, Vietnam that paves the way for improving the prophylaxis strategies for avian coccidiosis.
\end{abstract}

Keywords: Eimeria spp., ITS-1, Local chicken, Noi breed, PCR, Vietnam

\section{INTRODUCTION}

Poultry production is a crucial role in economic and social development in Vietnam. Intensive poultry husbandry farms are mainly located in urban areas near the two large delta regions, namely the Red River (North Vietnam) and the Mekong Delta (South Vietnam). The local chicken breeds occupied more than $70 \%$ of the total poultry population in Vietnam (Lan Phuong et al., 2015). Among them, the Noi breed is one of the most favorite breeds because of its high meat quality and being easily raised in many different environments, especially in Mekong Delta.

Coccidiosis, caused by the obligatory intracellular protozoan Eimeria spp., is one of the most common worldwide parasitic diseases, and it affects poultry production every year. Through the high morbidity, decreased efficiency of feed conversion, reduced egg production, diarrhea, and mortality (Allen and Fetterer 2002; Shirley et al., 2005; Blake and Tomley, 2014), coccidiosis causes a global economic loss of up to 3 billion dollars per year (Blake and Tomley, 2014). Furthermore, eradicating infectious oocysts shedding in the surrounding environment is challenging because the oocysts are of high persistency (Blake and Tomley, 2014) leading to reinfections in poultry. In the poultry industry, seven species of Eimeria, including E. tenella, E. maxima, $E$. necatrix, E. praecox, E. mitis, E. acervulina, and Eimeria brunetti can frequently appear in infected chickens, causing intestinal lesions with different severity. The infection rate of Eimeria was recorded in broilers in Red River Delta (North of Vietnam), Bac Giang, Hue city was $80.56 \%$ (Tran et al., 2014), 33.17\% (Doan et al., 2014), and 50.92\% (Huynh, 2017), respectively. However, there have been no reports on the prevalence of coccidiosis in the local chickens of Noi breed in the Mekong Delta, Vietnam. The current study, therefore, focused on evaluating the epidemiology of coccidiosis in Ben Tre and Hau Giang provinces in Mekong Delta by applying morphological and molecular techniques.

\section{MATERIALS AND METHODS}

\section{Ethical approval}

This current study was designed and performed based on Can Tho University regulations.

\section{Sample size}

The sample size was calculated based on the formula of Thrusfield (2007), $\mathrm{N}=\frac{1.96^{2} \times p q}{d^{2}}$ where, $\mathrm{N}$ presents the 
required sample size, $\mathrm{p}$ signifies expected prevalence, $\mathrm{q}$ refers to 1-p, and d explains desires absolute precision. The expected prevalence is assumed $50 \%$ because there has been no study on the prevalence of coccidiosis in local chickens of Noi breed in Ben Tre and Hau Giang provinces. With 5\% absolute precision at a 95\% confidence level and the expected prevalence at $50 \%$, the required samples were 384 . To increase the precision of the current study, 600 samples per province were collected. A total of 1200 samples were collected from 20 Noi breed chicken farms (free-ranging system, chickens aged 3-7 weeks) located in Ben Tre and Hau Giang provinces, Vietnam.

Vaccination against coccidiosis has not been administered to the surveyed chickens. The age and breed were informed by the owners. Fresh feces samples were randomly collected throughout the studied farms.

\section{Parasites preparation and microscopic examination}

Oocysts from fecal samples were collected by flotation method using saturated sodium chloride solution (Conway Donal and McKenzie, 2007). Clean oocysts were further identified based on oocysts morphometry (shape, size, color, the presence or absence of micropyle) and the sporulation time to differentiate Eimeria species, which were described by Levine (1985). All measurements were performed in 100 sporulated oocysts per species. The oocysts were visualized under microscope ECLIPSE Nikon 200. Then, oocysts and incubated in 2.5\% potassium dichromate solution $\left(\mathrm{K}_{2} \mathrm{Cr}_{2} \mathrm{O}_{7}\right)$ for $24-48 \mathrm{hr}$ at room temperature to observe the sporulation of oocysts. The number of coccidial oocysts in $1 \mathrm{~g}$ feces (OPG) was quantified by the McMaster method. The intensity of infection was classified into 4 levels based on OPG, namely $1+$ (less than 1000 oocysts), 2+ (1000-5000 oocysts), $3+$ (5000-20000 oocysts), and 4+ (more than 20000 oocysts)

\section{Identification of Eimeria species by the polymerase chain reaction}

DNA extraction was performed on Eimeria spp positive farms (5 samples per each). In this regard, $100 \mathrm{mg}$ of chicken feces were subjected to DNA extraction, using DNA Stool Kit (Qiagen, Germany) and following the instructions of the manufacturer.

\section{PCR amplification}

PCR targeting on internal transcribed spacer ITS-1 regions of rDNA was performed to detect different Eimeria species. Table 1 provides more detail about primer sequences, annealing temperature, and PCR product size. The single PCR reaction was performed, consisting of $12.5 \mu \mathrm{l}$ Master mix, $0.5 \mu \mathrm{l}$ of primer, $0.5 \mu \mathrm{l}$ dNTP $(10 \mu \mathrm{M})$, DNA/ nuclease-free water up to $25 \mu 1$. The thermal reaction cycling consists of initial denaturation with $96^{\circ} \mathrm{C}$ for 5 minutes, followed by 30 cycles of $95^{\circ} \mathrm{C}$ denaturations for 1 minute, $59-73^{\circ} \mathrm{C}$ annealing for 1 minute (depending on the Eimeria species); $72^{\circ} \mathrm{C}$ extensions for 1 minute 30 seconds, and a final extension $72^{\circ} \mathrm{C}$ for 7 minutes (Schnitzler et al., 1998; Lew et al., 2003). After amplification, PCR products were loaded on $1.5 \%(\mathrm{w} / \mathrm{v})$ agarose gel in $1 \mathrm{X}$ TAE $120 \mathrm{~V}$ in 30 min with a DNA ladder of $100 \mathrm{bp}$. The gel was stained by ethidium bromide and visualized under UV light (Biorad UV 2000)

Table 1. Species-specific primer sets for detecting Eimeria spp. by PCR

\begin{tabular}{|c|c|c|c|c|}
\hline & Eimeria spp & Primer sequences $\left(5^{\prime}-3^{\prime}\right)$ & $\begin{array}{l}\text { Annealing } \\
\text { temperature }\left({ }^{\circ} \mathrm{C}\right)\end{array}$ & $\begin{array}{l}\text { PCR product } \\
\text { size (bp) }\end{array}$ \\
\hline 1 & E. tenella & $\begin{array}{l}\text { ETF: ATTTTAGTCCATCGCACCCCT } \\
\text { ETR: CGAGGGCTCTGCATAGGACA }\end{array}$ & 59 & 278 \\
\hline 2 & E. acervulina & $\begin{array}{l}\text { EAF: GGCTTGGATGATGTTTGCTG } \\
\text { EAR: CGAACGCAATAACACACGCT }\end{array}$ & $62 *$ & 321 \\
\hline 3 & E. mitis & $\begin{array}{l}\text { EMi5FA: CGGAGCTGGGGTTTTCTTTC } \\
\text { EMi5RA: CTGCATATCCACAGTTCGAACATAC }\end{array}$ & $60 *$ & 193 \\
\hline 4 & E. maxima & $\begin{array}{l}\text { EMFA2: GCGGTTTCATCATCCATCATCG } \\
\text { EMRA2: CGTTGTGAGAAG/AACTGA/GAAGGG }\end{array}$ & $60 *$ & 145 \\
\hline 5 & E. necatrix & $\begin{array}{l}\text { ENF: TACATCCCAATCTTTGAATCG } \\
\text { ENR: GGCATACTAGCTTCGAGCAAC }\end{array}$ & 59 & 383 \\
\hline 6 & E. praecox & $\begin{array}{l}\text { EPRA: AAAAGCAACAGCGATTCAAG } \\
\text { EPRA: CCAAGCGATTTCATCATTCGGGGAG }\end{array}$ & 61 & 116 \\
\hline 7 & E. brunetti & $\begin{array}{l}\text { EBF: GATCAGTTTGAGCAAACCTTCG } \\
\text { EBR: TCTTCCGTACGTCGGAT }\end{array}$ & 73 & 311 \\
\hline
\end{tabular}




\section{Phylogenetic analysis}

The results of ITS-1 sequences were aligned using ClustalW Multiple alignments. Phylogenetic analysis was conducted using software MEGA X (The Molecular Evolution Genetics Analysis) and explained by the Maximum Likelihood method and Tamura-Nei model (Lew et al, 2003). Reference ITS-1 sequences of Eimeria from Sweden, Australia, India, and China isolates were accessed through GenBank (Table 2) and used in phylogenetic analysis.

Table 2. Sequences of ITS-1 used to perform phylogenetic analysis

\begin{tabular}{lll}
\hline GenBank accession no. & Species strain & Original \\
\hline AF446062.1 & E. mitis & Australia \\
AF026384.1 & E. acervulina & Sweden \\
AF446060.1 & E. maxima & Australia \\
KY117143.1 & E. tenella & China \\
GQ856312.1 & E. acervulina & India \\
GQ153626.1 & E. mitis & China \\
GQ856293.1 & E. maxima & India \\
AF446074.1 & E. tenella & Australia \\
\hline
\end{tabular}

\section{Data analysis}

The variations of infection prevalence and intensity among different age groups were determined by ChiSquare $(\chi 2)$ test and Tukey test using Minitab software (version 16). The significant difference was considered at $\mathrm{p}$ value $<0.05$.

\section{RESULTS}

The prevalence of coccidiosis in survey areas in the Mekong Delta, Vietnam

The overall infection rate of coccidiosis in local chickens (Noi breed) in Ben Tre and Hau Giang provinces was $67.82 \%$ and $68.50 \%$, respectively (Tables 3 and 4 ). In Ben Tre province, the prevalence of coccidiosis in local chickens in Ben Tre province tended to increase by age. The highest infection rate and intensity $(4+)$ were recorded at 6 -week-old chickens with $100 \%$ as $84.2 \%$, respectively. There was a statistically significant infection rate among age groups in Ben Tre $(\chi 2=260.26, \mathrm{df}=4, \mathrm{p}<0.05)$ and in Hau Giang $\left(\chi_{2}=77.51, \mathrm{df}=4, \mathrm{p}<0.05\right)$. The infection rate of coccidiosis in Hau Giang province also increased from the third week to the fourth week; however, the infection rate significantly declined in next following week ( $\mathrm{p}<0.05)$. At the fourth week, the peak of infection was recorded with an infection rate of $96.67 \%$ and the intensity (4+) with $25.86 \%$, resulting in clinical symptoms of coccidiosis, such as anorexia, foamy feces, and bloody droppings.

\section{Morphological and molecular identification}

The morphological and molecular identification was carried on 60 samples from 12 positive farms (5 samples/ farm). Based on the morphological features of Eimeria spp. oocysts and sporulation time, the characteristics of each Eimeria species were described in detail (Table 5 and figure 1). In the current study, five different Eimeria species E. mitis E. necatrix, E. acervulina, E. maxima, and E. tenella, were found in fecal samples of chickens in the Mekong Delta. However, we determined four Eimeria species except for E. necatrix by PCR method. E. praecox and $E$. brunetti were not detected from tested samples in both methods. By applying molecular technique, E. acervulina, E. maxima, E. mitis, and E. tenella were successfully amplified with corresponding amplicons 321 bp, 145bp, 193 bp, and 278bp (Figure 2). The prevalence rates of E. mitis, E. acervulina, E. maxima, and E. tenella were $20 \%, 61.67 \%, 66.66 \%$, and $83.33 \%$, respectively

\section{Phylogenetic analysis}

The Maximum Likelihood method was used to generate the phylogenetic tree, based on the ITS-1 sequences from Eimeria species from local chicken (Noi breed) in the current study and other ITS-1 sequences of Eimeria species available in the GenBank database from Australia, Sweden, China, and India (Table 2). BLAST analysis of nucleotide sequences of E. mitis, E. acervulina, E. maxima, and E. tenella in the current study showed high homology (95.54\% to $98.97 \%)$ with corresponding Eimeria strains deposited in GenBank (GQ153626.1, AF026384.1, AF446060.1, KY117143.1) The phylogenetic tree demonstrated that E. tenella had close relationships to E. mitis and E. acervulina (Figure 3). However, E. maxima sequences were clustered into two different clades. E. maxima isolate in Vietnam and Australia formed one small cluster and separated to GQ85629 E. maxima isolate from India. 
Table 3. The infection rate and infection intensity of coccidiosis in Ben Tre province, Vietnam, by microscopic examination

\begin{tabular}{|c|c|c|c|c|c|c|c|}
\hline \multirow{3}{*}{ Age (week) } & \multirow{2}{*}{\multicolumn{3}{|c|}{ Infection rate }} & \multicolumn{4}{|c|}{ Intensity of infection and percentage } \\
\hline & & & & $1+$ & $2+$ & $3+$ & $4+$ \\
\hline & Samples & Positive samples & Percentage $(\%)$ & $(\%)$ & $(\%)$ & $(\%)$ & $(\%)$ \\
\hline 3 & 120 & 25 & $20.8^{\mathrm{a}}$ & 60 & 40 & 0 & 0 \\
\hline 4 & 120 & 51 & $42.5^{\mathrm{b}}$ & 52.9 & 39.2 & 5.88 & 1.96 \\
\hline 5 & 120 & 100 & $83.3^{\mathrm{c}}$ & 14 & 25 & 37 & 24 \\
\hline 6 & 120 & 120 & $100^{\mathrm{d}}$ & 0 & 5.8 & 10 & 84.2 \\
\hline 7 & 120 & 111 & $92.5^{\mathrm{e}}$ & 36 & 25.2 & 12.6 & 26.1 \\
\hline Total & 600 & 407 & 67.83 & 40.73 & 27.04 & 16.37 & 34.06 \\
\hline
\end{tabular}

$\overline{\mathrm{a}, \mathrm{b}, \mathrm{c}, \mathrm{d}, \mathrm{e}}$ Values in the same column with different superscripts differ significantly $(\mathrm{p}<0.05)$.

Table 4. The infection rate and infection intensity of coccidiosis in Hau Giang province, Vietnam, by microscopic examination

\begin{tabular}{|c|c|c|c|c|c|c|c|}
\hline \multirow{3}{*}{ Age (week) } & \multirow{2}{*}{\multicolumn{3}{|c|}{ Infection rate }} & \multicolumn{4}{|c|}{ Intensity of infection and percentage } \\
\hline & & & & \multirow{2}{*}{$\begin{array}{c}1+ \\
(\%) \\
\end{array}$} & \multirow{2}{*}{$\begin{array}{c}2+ \\
(\%) \\
\end{array}$} & \multirow{2}{*}{$\begin{array}{c}3+ \\
(\%) \\
\end{array}$} & \multirow{2}{*}{$\begin{array}{c}4+ \\
(\%) \\
\end{array}$} \\
\hline & Samples & Positive samples & Percentage $(\%)$ & & & & \\
\hline 3 & 120 & 67 & $55.83^{\mathrm{a}}$ & 58.21 & 22.39 & 7.46 & 11.94 \\
\hline 4 & 120 & 116 & $96.67^{\mathrm{b}}$ & 24.14 & 26.72 & 23.28 & 25.86 \\
\hline 5 & 120 & 89 & $74.17^{\mathrm{c}}$ & 30.34 & 30.34 & 22.47 & 16.85 \\
\hline 6 & 120 & 81 & $67.5^{\mathrm{c}}$ & 38.27 & 33.33 & 18.52 & 9.88 \\
\hline 7 & 120 & 58 & $48.33^{\mathrm{a}}$ & 58.62 & 15.52 & 17.24 & 8.62 \\
\hline Total & 600 & 411 & 68.5 & 41.92 & 25.66 & 17.79 & 14.63 \\
\hline
\end{tabular}

${ }^{\mathrm{a}, \mathrm{b}, \mathrm{c}, \mathrm{d}}$ Values in the same column with different superscripts differ significantly $(\mathrm{p}<0.05)$.
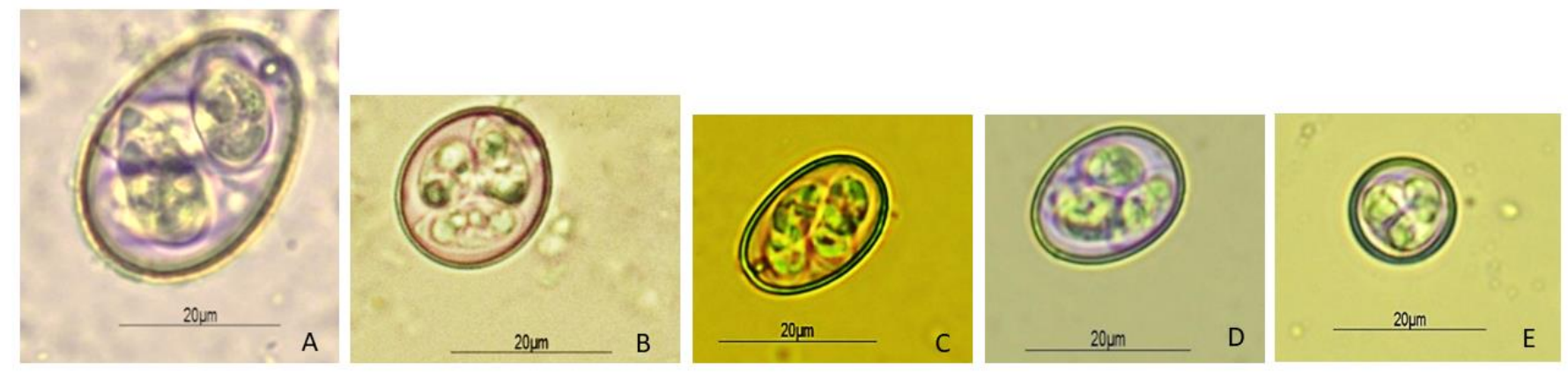

Figure 1. Microscopic images of Eimeria species. A: E. maxima, B: E. tenella, C: E. necatrix, D: E. acervulina, E: E. mitis

Table 5. Morphological characteristics of Eimeria species

\begin{tabular}{lllll}
\hline Oocyst characteristics & $\begin{array}{l}\text { Length }(\boldsymbol{\mu m}) \\
\text { Mean } \pm \mathbf{S E}\end{array}$ & $\begin{array}{l}\text { Width }(\boldsymbol{\mu m}) \\
\text { Mean } \pm \mathbf{S E}\end{array}$ & $\begin{array}{l}\text { Sporulation time } \\
(\mathbf{h})\end{array}$ & Species \\
\hline $\begin{array}{l}\text { Oocyst ovoid or ellipsoid, } \\
\text { smooth wall, no micropyle }\end{array}$ & $18.08 \pm 0.641$ & $14.24 \pm 0.78$ & $16-28$ & E. acervulina \\
\hline $\begin{array}{l}\text { Oocyst ovoid, yellowish, slightly rough } \\
\text { surface wall, without micropyle }\end{array}$ & $31.83 \pm 1.145$ & $21.29 \pm 0.99$ & $24-42$ & E. maxima \\
\hline $\begin{array}{l}\text { Oocyst spherical, smooth and } \\
\text { colourless wall, no micropyle }\end{array}$ & $16.07 \pm 0.883$ & $15.43 \pm 0.889$ & $14-36$ & E. mitis \\
\hline $\begin{array}{l}\text { Oocyst ovoid, smooth and } \\
\text { colourless wall, no micropyle }\end{array}$ & $23.48 \pm 0.83$ & $19.29 \pm 0.75$ & $18-26$ & E. tenella \\
\hline $\begin{array}{l}\text { Oocyst ovoid or spherical, } \\
\text { smooth wall, no micropyle }\end{array}$ & $18.84 \pm 1.41$ & $16.40 \pm 1.54$ & $20-32$ & E. necatrix \\
\hline
\end{tabular}

Measurement was conducted in 100 oocysts for each Eimeria species. SE: Standard errors 

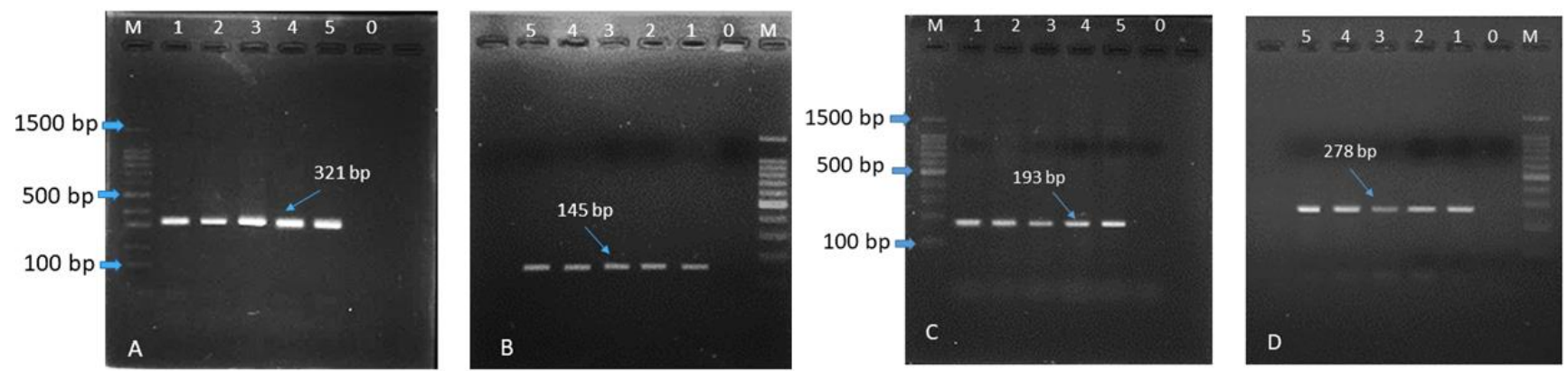

Figure 2. PCR results of the Eimeria species-specific ITS-1 region, followed by agarose gel $2 \%$ electrophoresis. M: Marker 100 bp, Lane 0: negative control, and lanes 1, 2, 3, 4, 5 samples. A: E. acervulina 321 bp, B: E. maxima 145 bp, C: E. mitis 193 bp, D: E. tenella $278 \mathrm{bp}$.

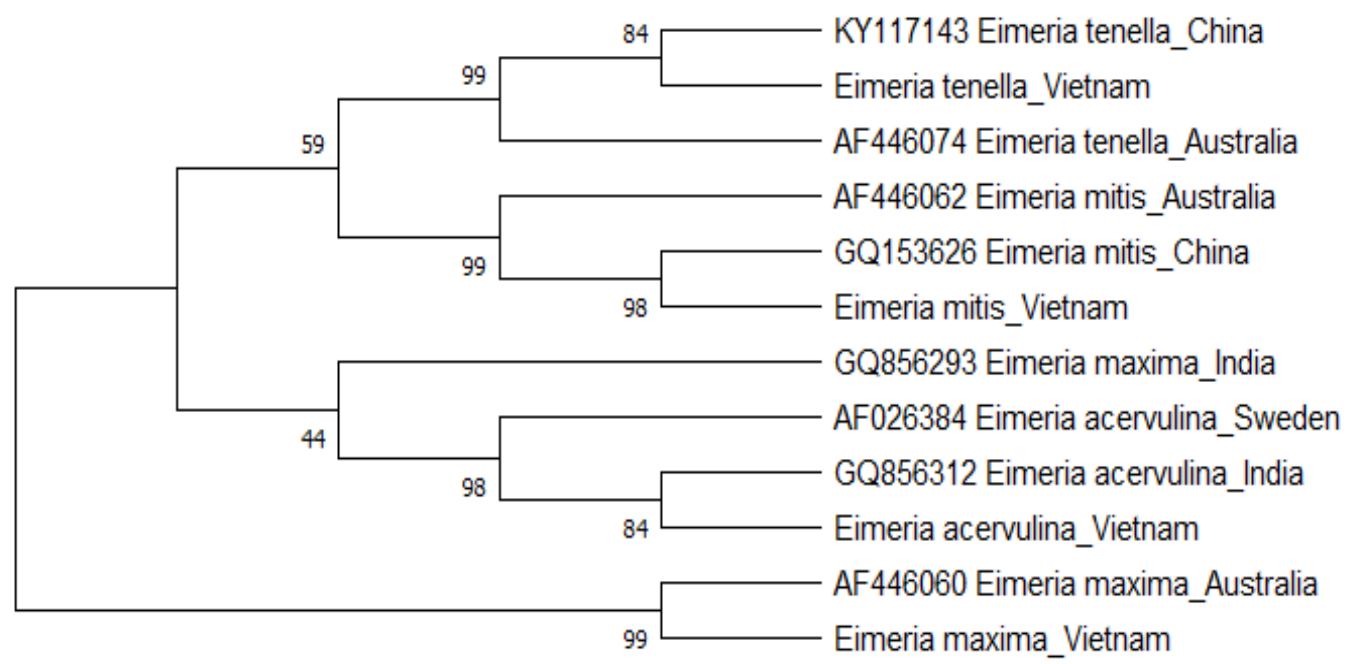

Figure 3. Phylogenetic analysis based on ITS-1 nucleotide sequences of Eimeria spp. using the Maximum Likelihood method (Tamura-Nei model) with bootstrap 1000 replications.

\section{DISCUSSION}

Chickens are a crucial source of food supplies in Vietnam. However, the avian coccidiosis causes dramatic economic loss due to high mortality, reduced nutrient absorption, growth retardation, and decreased productivity of poultry. Besides commercial broilers, local chickens also play an important role to supply meat for local markets but the coccidiosis in local chickens especially in the Noi breed is neglected. The overall infection rate of coccidiosis in the local chicken- Noi breed was higher than studies in broilers in Bac Giang province $33.17 \%$ (Doan et al., 2014) and Thua Thien Hue province 50.92\% (Huynh, 2017). The current study showed that the prevalence of coccidiosis in the local chicken- Noi breed was influenced by age. The high infection rate was seen in chickens from weeks 4 to 6. This might be explained due to the dramatic decrease of maternal immunity against coccidiosis in during 31-45 days (Kaboudi et al., 2016). Moreover, the accumulation of oocysts in the poultry litter on the ground created good opportunities for reinfection. These findings are accordant to studies as indicating that poultry had a high prevalence of coccidiosis during 31-45 days (Sharma et al., 2015) (Kaboudi et al., 2016). Moreover, the high prevalence also revealed that the prophylaxis strategies of coccidiosis in surveyed farms were not adequately concerned. Noi breed chickens were reared in a free-ranging system and were not vaccinated for coccidiosis. The farmers just gave anticoccidial drugs as observing the typical symptoms such as anorexia, foamy feces, and bloody droppings. This situation was also recorded in many farms in Hau Giang province, resulting in a significant decrease in the prevalence of coccidiosis from the fourth to fifth week. However, the findings of the present study were lower than studies in Red River Delta, Vietnam as $80.56 \%$ (Tran et al., 2014), in An Hui, China, as $87.75 \%$ (Huang et al., 
2017), and in Columbia as 92.8\% (Mesa et al., 2021). The possible explanation can be related to risk factors, such as age, breed, husbandry system, and disease management that can result in the various prevalence rates of coccidiosis.

E. tenella, E. maxima, E. acervulina, and E.mitis were found in both morphological and molecular methods. $E$. necatrix has been found by morphological identification in the current study, but it was not confirmed by molecular technique. It was a challenge in the morphological identification of $E$. necatrix due to the overlap in morphological characteristics between E. tenella and $E$. necatrix that was also mentioned in the study of Matsubayashi et al., (2020). Moreover, the mixed infection with many Eimeria species occurred more frequently than a single infection (Carvalho et al., 2011) that also hindered the accurately morphological identification. The current study revealed that it is necessary to apply molecular techniques in Eimeria species identification. In fact, the precise identification of Eimeria species plays an important role in diagnosing disease and developing effective treatments, or prophylaxis in live vaccines (Aarthi et al., 2010) and biological studies (Lee et al., 2010). Thereby, the molecular identification based on many gene targets, such as small subunit rRNA (Tsuji et al., 1997), ITS-1 (Schnitzler et al. 1998; Lew et al., 2003; Kumar et al., 2014) has been established to differentiate species of Eimeria to overcome the limitations of morphological identification. Among them, ITS-1 sequences have been considered as a genetic marker for the identification of species, including Eimeria species due to high heterogeneity among different species. By PCR targeting ITS-1, the prevalence of E. tenella, E. maxima, E. acervulina, and E. mitis was $83.33 \%, 66.66 \%, 61.67 \%$, and $20.00 \%$, respectively. These findings are in line with many earlier studies in Bac Giang 78.16\% (Doan et al., 2014), in Red River Delta in Vietnam 90\% (Tran et al., 2014) indicating that E. tenella is the predominant Eimeria spp. in domestic fowl in Vietnam and worldwide. Besides, E. tenella is one of the most pathogenicity leading to clinical symptoms in chickens. Thus, it is necessary to have appropriate prophylaxis strategies in this survey area to minimize economical loss.

The phylogenetic analysis of the ITS-1 sequences from Eimeria species showed a clear species-specific cluster, regardless of the geographical distribution for all ITS-1 sequences of E. tenella, E. acervulina, and E. mitis. However, E. maxima in Vietnam and Australia formed a cluster and was separated from E. maxima in India. This could be explained due to the diversity of intra-species
ITS-1 lineages of E. maxima, which was reported in previous studies (Lew et al., 2003; Cantacessi et al., 2008; Kumar et al., 2015). Understanding the occurrence of Eimeria species in specific regions using molecular detection methods is useful for choosing an appropriate vaccine to prevent coccidiosis.

\section{CONCLUSION}

The high prevalence of coccidiosis in local chickens (Noi breed) in the Mekong Delta revealed that the disease management was not adequately performed. Therefore, the farmers should pay attention to improving environmental hygiene and prophylaxis strategies in local chickens to prevent avian coccidiosis outbreaks in Vietnam and minimize economic loss. In addition, the study characterized the molecular information about four Eimeria species in the Noi breed, including $E$. tenella, E. acervulina, E. maxima, and $E$. mitis. The findings can be of great contribution to future studies for establishing appropriate prophylaxis strategies, especially in developing the coccidiosis vaccine in Vietnam. This study was one of the first attempts to apply molecular techniques to characterize Eimeria species in the local chicken breed (Noi) in Mekong Delta, Vietnam.

\section{DECLARATION}

\section{Authors' contribution}

Hung Nguyen Huu, and Tran Nguyen-Ho-Bao designed the study, data analysis, wrote the manuscript with the contribution from Trung Van Le, Tien Ai Lu. All authors established experiment protocols.

\section{Acknowledgment}

The authors sincerely acknowledge the assistance of Tran Van Be Nam in Biotechnology Research and Development Institute at Can Tho University. We would like to thank all veterinarians and farmers for their support in collecting samples in surveyed areas. The current study is funded in part by the Can Tho University Improvement Project VN14-P6 (Program A10) supported by a Japanese ODA loan.

\section{Competing interests}

The authors declare that they have no competing interests

\section{Ethical considerations}

Ethical issues including data fabrication and/ or falsification, plagiarism, consent to publish, double submission, redundancy) have been carefully checked by the authors. 


\section{REFERENCES}

Aarthi S, Dhinakar Raj G, Raman M, Gomathinayagam S, and Kumanan K (2010). Molecular prevalence and preponderance of Eimeria spp. among chickens in Tamil Nadu, India. Parasitology Research, 107: 1013-1017. DOI: https://www.doi.org/10.1007/s00436-010-1971-2

Allen P, and Fetterer R (2002). Recent advances in biology and immunobiology of Eimeria species and in diagnosis and control of infection with these coccidian parasites of poultry. Clinical Microbiology Reviews, 15: 58-65. DOI: https://www.doi.org/10.1128/CMR.15.1.58-65.2002

Blake DP, and Tomley FM (2014). Securing poultry production from the ever-present Eimeria challenge. Trends Parasitol, 30: 12-19. DOI: https://www.doi.org/10.1016/j.pt.2013.10.003

Cantacessi C, Riddell S, Morris GM, Doran T, Woods WG, Otranto D, and Gasser RB (2008). Genetic characterization of three unique operational taxonomic units of Eimeria from chickens in Australia based on nuclear spacer ribosomal DNA. Veterinary Parasitology, 152: 226-234. DOI: https://www.doi.org/10.1016/j.vetpar.2007.12.028

Carvalho FS, Wenceslau AA, Teixeira M, and Albuquerque GR (2011). Molecular diagnosis of Eimeria species affecting naturally infected Gallus gallus. Genetics and Molecular Research, $\quad$ 10: 996-1005. DOI: https://www.doi.org/10.4238/vol10-2gmr1043

Conway Donal P, and McKenzie E (2007). Poultry coccidiosis: Diagnosis and testing procerdure, 3rd edition. Blackwell Publishing. Available at: https://www.wiley.com/enus/Poultry+Coccidiosis\%3A+Diagnostic+and+Testing+Proc edures\%2C+3rd+Edition-p-9780470344323

Doan TT, Tran ĐH, Nguyen, Huu Nam, and Nguyen THC (2014). Survey of coccidiosis in chickens in Bac Giang province. Veterinary Science, 21: 68-75.

Huang Y, Ruan X, Li L, and Zeng M (2017). Prevalence of Eimeria species in domestic chickens in Anhui province, China. Journal Parasitic Diseases, 41: 1014-1019. DOI: https://www.doi.org/10.1007/s12639-017-0927-1

Huynh VC (2017). Survey on some pathological features of coccidiosis in chickens and research on manufacturing probiotics for use in prevention and treatment. $\mathrm{PhD}$ dissertation. Vietnam National University of Agriculture.

Kaboudi K, Umar S, Tanveer M, and Munir (2016). Prevalence of Coccidiosis in Free-Range Chicken in Sidi Thabet, Tunisia. Scientifica (Cairo), Article ID 7075195. DOI: https://www.doi.org/10.1155/2016/7075195

Kumar S, Garg R, Moftah A, Clark EL, Macdonald SE, Chaudhry AS, Sparagano O, Banerjee PS, Kundu K, Tomley FM et al. (2014). An optimised protocol for molecular identification of Eimeria from chickens. Veterinary Parasitology, 199: 24-31. DOI: https://www.doi.org/10.1016/j.vetpar.2013.09.026

Kumar S, Garg R, Banerjee PS, Ram H, Kundu K, Kumar S, and Mandal M (2015). Genetic diversity within ITS-1 region of Eimeria species infecting chickens of North India. Infection Genetics Evolution, 36: 262-267. DOI: https://www.doi.org/10.1016/j.meegid.2015.09.023

Lan Phuong T, Dong Xuan K, and Szalay I (2015). Traditions and local use of native Vietnamese chicken breeds in sustainable rural farming. Worlds Poultry Science Journal, 71: 385-396. DOI: https://www.doi.org/doi:10.1017/S0043933915000380

Lee BH, Kim WH, Jeong J, Yoo J, Kwon YK, Jung BY, Kwon JH, Lillehoj HS, and Min W (2010). Prevalence and crossimmunity of Eimeria species on Korean chicken farms. Journal of Veterinary Medical Science, 72(8): 985-989. DOI: https://www.doi.org/10.1292/jvms.09-0517

Levine N (1985). Veterinary protozoology, 1st edition. Iowa State University Press

Lew AE, Anderson GR, Minchin CM, Jeston PJ, and Jorgensen WK (2003). Inter- and intra-strain variation and PCR detection of the internal transcribed spacer 1 (ITS-1) sequences of Australian isolates of Eimeria species from chickens. Veterinary Parasitology, 112: 33-50. DOI: https://www.doi.org/10.1016/S0304-4017(02)00393-X

Matsubayashi M, Shibahara T, Matsuo T, Hatabu T, Yamagishi J, Sasai K, and Isobe T (2020). Morphological and molecular identification of Eimeria spp. in breeding chicken farms of Japan. Journal of Veterinary Medicine Science, 82: 516-519. DOI: https://www.doi.org/10.1292/jvms.19-0661

Mesa C, Gómez-Osorio LM, López-Osorio S, Williams SM, and Chaparro-Gutiérrez JJ (2021). Survey of coccidia on commercial broiler farms in Colombia: frequency of Eimeria species, anticoccidial sensitivity, and histopathology. Poultry Science, 100(8): 101239. DOI: https://www.doi.org/10.1016/j.psj.2021.101239.

Schnitzler BE, Thebo PL, Mattsson JG, Tomley FM, and Shirley MW (1998). Development of a diagnostic PCR assay for the detection and discrimination of four pathogenic Eimeria species of the chicken. Avian Pathology, 27: 490-497. DOI: https://www.doi.org/10.1080/03079459808419373

Sharma S, Iqbal A, Azmi S, Mushtaq I, Wani ZA, and Ahmad S (2015). Prevalence of poultry coccidiosis in Jammu region Kashmir State. Journal Parasitic Diseases, 39: 85-89 DOI: https://www.doi.org/10.1007/s12639-013-0286-5

Shirley MW, Smith AL, and Tomley FM (2005). The biology of avian Eimeria with an emphasis on their control by vaccination. Advances in Parasitoly, 60: 285-330. DOI: https://www.doi.org/10.1016/S0065-308X(05)60005-X

Thrusfield M (2007). Veterinary epidemiology. 3rd edition. John Wiley and Sons, Scotland. Available at: https://www.wiley.com/en-

au/Veterinary+Epidemiology,+3rd+Edition-p9781118713419

Tran HD, Gadahi JA, and Leghari RA (2014). Molecular identification of Eimeria species Infection in chickens in surrounding areas of the red river delta in Vietnam. International Journal of Livestock Research, 4: 9. DOI: https://www.doi.org/10.5455/ijlr.20140925111501

Tsuji N, Kawazu S, Ohta M, Kamio T, Isobe T, and Shimura K, and Fujisaki K (1997). Discrimination of eight chicken Eimeria species using the two-step polymerase chain reaction. Journal of Parasitology, pp. 966-970. Available at: https://www.jstor.org/stable/3284302 